\title{
XXXVI. On the law of molecular force
}

\section{William Sutherland M.A. B.Sc.}

To cite this article: William Sutherland M.A. B.Sc. (1889) XXXVI. On the law of molecular force, Philosophical Magazine Series 5, 27:167, 305-321, DOI: 10.1080/14786448908628359

To link to this article: http://dx.doi.org/10.1080/14786448908628359

$$
\text { 册 Published online: } 29 \text { Apr } 2009 .
$$

Submit your article to this journal

Џ Article views: 9

Q View related articles ¿ 


\section{$\left[\begin{array}{lll}3 & 305\end{array}\right]$}

\section{On the Law of Molecular Force. By William Sutherland, M.A., B.Sc.*}

TN the Philosophical Magazine for August 1886, and July and August 1887, I advanced the hypothesis that the law of molecular force is that of the inverse fourth power of the distance, and considered how far its consequences harmonize with the results of the experiments of Thomson and Joule on the expansion of gases through porous plugs, and with the experiments of Amagat and Andrews on the volume occupied by $\mathrm{CO}_{2}$ at different temperatures and pressures. In the present paper I shall discuss the same law in the light of the recent beautiful discoveries of Eötvös and Robert Schiff in capillarity, and shall show, as the chief result of the inquiry, the following law of the parameter $\mathrm{A}$ in the expression $3 \mathrm{Am}^{2} / r^{4}$, which expresses the force between two similar molecules of mass $m$ at distance $r$ apart, the law of their mutual potential being $-\frac{\mathrm{A} m^{2}}{r^{3}}$.

In compounds containing $\mathrm{C}, \mathrm{O}$, and $\mathrm{H}$ the molecule may be considered to have a volume, to which each atom of $\mathrm{H}$ contributes an amount very small in comparison with that contributed by an atom of $\mathrm{O}$ and of $\mathrm{C}$; while an atom of $\mathrm{O}$, when singly bound to another atom, contributes an amount equal to that of two carbon atoms, and when doubly bound equal to that of three carbon atoms. The volume of such a molecule can then be expressed in terms of that of a carbon atom, and the parameter $\mathrm{A}$ varies inversely as the surface of the molecule.

By the volume of a molecule I do not mean what is usually called the molecular volume (an objectionable term, which I would propose to replace by the term molecular domain), but the actual volume of the molecule. Another result of the investigation will be to show that the rate of change of the translatory kinetic energy of nearly all liquid molecules with temperature is the same when measured at low constant pressure.

Eötvös (Wiedemann, xxvii.) announces the following remarkable law :-The rate of variation with temperature of the product of the surface-tension of a liquid by its molecular domain raised to the power two thirds, is the same for all liquids; or, in symbols, if $\alpha$ denote surface-tension, and $v$ molecular domain,

$$
\frac{d}{d t}\left(\alpha v^{\frac{2}{3}}\right)=\cdot 227 .
$$

* Communicated by the Author.

Phil. Mag. S. 5. Vol. 27. No. 167. April 1889. X 
He found this to hold for a large number of liquids ; and also that water, the alcohols, and the fatty acids are exceptional at the lower ranges of temperature. The theoretical grounds on which he founds the law are, in the present state of physics, somewhat transcendental ; but as they illustrate the power of the idea of " correspondence," which Van der Waals has developed so brilliantly, they can be reproduced here with advantage.

Let $v$ be the domain of a molecule when it is part of a mass of liquid, and $u$ when part of a mass of gas at the same temperature as the liquid; then, if the temperatures $\mathrm{T}_{1}$ and $\mathrm{T}_{2}$ for two substances are chosen so that $v_{1} / u_{1}=v_{2} / u_{2}$, the conditions of the molecules are considered by Eötvös to "correspond." This equation may be written

$$
v_{1} p_{1} / \mathrm{T}_{1}=v_{2} p_{2} / \mathrm{T}_{2} \text {; }
$$

where $p_{1}$ and $p_{2}$ are the pressures of saturation at the temperatures $T_{1}$ and $T_{2}$, if we consider the vapours of the liquids as approximately perfect gases. Then, according to the method of "correspondence," it is stated that the forces acting on the two molecules and the energies of the two molecules correspond. Consider a plane area over which $n$ molecules are distributed. The pressure on it is $n p_{1} v_{1}^{\frac{2}{3}} ;$; the tension across a line in the surface-film on which $l$ molecules lies is $l v_{1}^{\frac{1}{3}} \alpha_{1}$, where $\alpha_{1}$ is the surface-tension or surface-energy. Then, from the assumption as to complete correspondence in the states of the two molecules, we have

$$
\frac{l v_{1}^{\frac{1}{3}} \alpha_{1}}{n p_{1} v_{1}^{\frac{2}{3}}}=\frac{l v_{2}^{\frac{1}{3}} \alpha_{2}}{n p_{2} v_{2}^{\frac{2}{3}}}, \quad \text { or } \frac{\alpha_{1}}{p_{1} v_{1}^{\frac{1}{3}}}=\frac{\alpha_{2}}{p_{2} v_{2}^{\frac{1}{3}}} . \text {. . }
$$

Arguing in a similar manner as to correspondence in the change of energy of the two molecules to their surface-energy when they evaporate with absorption of latent heats $m_{1} \mathrm{~L}_{1}$, $m_{2} \mathrm{~L}_{2}$ respectively, $n_{1}$ and $m_{2}$ being the molecular weights, Eötvös writes

$$
\frac{m_{1} \mathrm{~L}_{1}}{\alpha_{1} v_{1}^{\frac{2}{3}}}=\frac{m_{2} \mathrm{~L}_{2}}{\alpha_{2} v_{2}^{\frac{2}{3}}} \text {. . . . . . . }
$$

This last result is known as Waterston's law (Phil. Mag. 1857, vol. xiv.), announced by him without reference to the idea of correspondence ; but, according to Eötvös, the quantities $\mathrm{L}_{1}$, $\alpha_{1}, v_{1}, \mathrm{~L}_{2}$, \&c. must be taken only for corresponding states of the two bodies. We shall see afterwards that Waterston's law cannot be regarded as an accurate physical law when tested by the available experimental material ; but that, as a rough statement of a connexion between surface-tension and latent heat, it can be deduced from the law of the inverse fourth power. 
Combining equations (1) and (2), we get

$$
\frac{\alpha_{1} v_{1}^{\frac{2}{3}}}{\mathrm{~T}_{1}}=\frac{\alpha_{2} v_{2}^{\frac{2}{3}}}{\mathrm{~T}_{2}^{1}} \text {. . . . . . . . }
$$

Now, according to Van der Waals, if two bodies are in corresponding states at temperatures $T_{1}$ and $T_{2}$, they will continue to be so at temperatures $(1+n) \mathrm{T}_{1}$ and $(1+n) \mathrm{T}_{2}$; in the case where $n$ is an infinitely small fraction we get from (4),

i. e.

$$
n \mathrm{~T}_{1} \frac{d}{d \mathrm{~T}}\left(\frac{\alpha_{1} v_{1}^{\frac{2}{3}}}{\mathrm{~T}_{1}}\right)=n \mathrm{~T}_{2} \frac{d}{d \mathrm{~T}}\left(\frac{\alpha_{2} v_{2}^{\frac{2}{3}}}{\mathrm{~T}_{2}}\right)
$$

$$
\frac{d}{d t}\left(\alpha_{1} v_{1}^{\frac{2}{3}}\right)=\frac{d}{d t}\left(\alpha_{2} v_{2}^{\frac{2}{3}}\right)=\text { constant. }
$$

Thus, according to this argument the last expression is not only constant for a given body at all temperatures, but is the same for all bodies. Eötvös has verified the formula from $0^{\circ}-190^{\circ}$ for ethyl oxide, and has found the mean value of the constant for a number of substances to be 2227 .

The exceptional behaviour of water, the alcohols, and the fatty acids is easily understood when we remember the large amount of experimental evidence there is pointing to irregularity in the molecular structure of water and ethyl alcohol, while the well-known anomalous vapour-density of acetic acid and of some of the higher acids of the series helps to explain their exceptional behaviour.

Robert Schiff's discovery (Annalen der Chemie, ccxxiii.) was the result of a comprehensive experimental determination of the capillary constants of a large number of organic compounds, and consisted in the unfolding of a definite relation between the number of molecules of different liquids, which rise in a given capillary tube, and their chemical structure. He showed that the number of molecules raised could be expressed as a number of hydrogen atoms ; thus the presence of a $\mathrm{C}$ atom in a molecule has as much influence in determining how many molecules will rise in a tube as two $\mathrm{H}$ atoms, an $\mathrm{O}$ atom as much as three $\mathrm{H}$, and so on. Calling the capillary equivalent of a molecule thus expressed in terms of $H$ atoms $h$, and $n 1000$ times the number of molecules raised, or $1000 \frac{a^{2} d}{2 m}$, where $a^{2}$ is the height to which the substance rises in a millimetre tube, $d$ its density, and $m$ its molecular weight, Schiff's empirical law is

$$
\log _{10} n=2 \cdot 8155-\cdot 00728 h-\log _{10} h .
$$

We shall not have occasion to use this result of Schiff's; but $\mathrm{X} 2$ 
his rich experimental material, published in the Annalen der Chemie, vol. cexxiii., and in Wiedemann's Beiblätter, vol. ix. (from the Mem. della $R$. Acc. $d$. Lincei, 1884), combined with Eötvös' law, will be used to determine the parameter A ; it is therefore necessary briefly to take note of a discussion which has been raised over a certain assumption of Schiff"s. $\mathrm{H}_{\theta}$ assumes that the angle of contact of liquid with glass is zero in every case in his determinations; but at the same time, in order to take account of the weight of the meniscus in his determination of surface-tension, he gives measurements of the distance between the top and the bottom of the meniscus, as this distance is in many cases different from the radius of the tube. The meniscus surface would appear to be different from the hemisphere, which it ought very approximately to be if the contact-angle were zero. Volkmann pointed out this (Annalen der Chemie, vol. cexxviii.), and recalculated the capillary constants from Schiff's determinations on the assumption that the data for the height of meniseus might be used for determining the angle of contact which he introduces into the calculation for the surface-tension. These recalculations, while they take away from the definiteness of some of Schiff's minor results, do not affect the truth of his broad discovery. There is no doubt that, on theoretical grounds, Volkmann's criticism is sound enough; that the experimental data, as furnished, bore evidence to the existence of a finite contactangle not taken into account in the calculations. But it must be remembered that the measurements of the heights of the meniscus were undertaken only for use in a small correction; and that such measurements are not competent to give satisfactory evidence of a finite contact-angle, and are certainly not competent to give a measure of it. The manner in which the apparent height of the meniscus varies in Schiff's data with temperature shows in a practical manner the unreliability of such measurements for testing the difficult question of contactangle. Schiff admits the theoretical justness of Volkmann's criticism as to contact-angle, but appeals to the regularity of his own results as his practical justification. As Volkmann himself has shown (and Quincke also) that the contact-angle for a large number of watery solutions of inorganic salts is zero, and as ordinary meniscus measurements would not demonstrate the fact, we are on the whole safe in assuming with Schiff that all his liquids wet glass (that is, that their contactangle is zero), until the point is settled by a satisfactory experimental method.

In applying the law of the inverse fourth power to the capillary theory we cannot use directly the formulæ already 
developed in Laplace's theory, seeing that there are assumptions involved in them which are not required or permissible with the law of the inverse fourth power.

For the purposes of this paper it will suffice to consider the surface-tension in a meniscus in a circular tube of so small diameter that its surface may be regarded as portion of a sphere, not necessarily a hemisphere, as we must provide for the case where the contact-angle is not zero.

We desire to find the force exerted by the meniscus A P B C P D (fig. 1) on a column of water, $P Q$, of small section lying along the axis of the tube. 'The meniscus consists of two parts : one, the surface layer, of variable density, which is enclosed between the two surfaces $\mathrm{APB}$ and $\mathrm{A}^{\prime} \mathrm{P}^{\prime} \mathrm{B}^{\prime}$; let the mean density in this layer be $\bar{\rho}$, while $\rho$ is the density in the body of the fluid. The other part of the meniscus $\mathrm{A}^{\prime} \mathrm{ED}, \mathrm{B}^{\prime} \mathrm{FC}$ is therefore of density $\rho$; but we

Fig. 1.

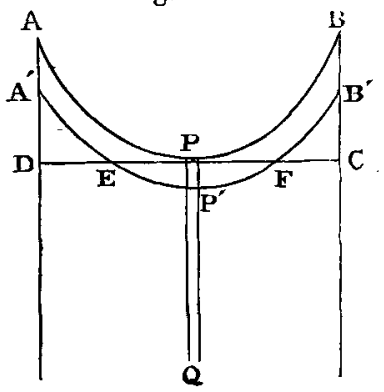
may regard the whole meniscus as of density $\bar{\rho}$, seeing that the parts $\mathrm{A}^{\prime} \mathrm{E} D$ and $\mathrm{B}^{\prime} \mathrm{F} C$ will contribute a small part of the whole force. In the same way we can regard the column P Q as throughout of density $\bar{\rho}$, seeing that the force exerted on $\mathrm{P}^{\prime} \mathrm{Q}$ is a small part of the whole. Now, for purposes of integration, we wish to replace the discontinuous distribution of molecules in the meniscus and column by an equivalent uniform distribution of matter. Accordingly, as I pointed out in a previous paper, we must be careful to provide a certain distance between our continuous meniscus and the base of the continuous column. This distance is not necessarily the same as the mean diameter of the molecular domain, but is a quantity of the same order of magnitude. The problem then reduces itself to the determination of the resultant attraction of a meniscus A PBCPD (fig. 2), the centre of whose surface is at $O$, on a column $Q T$, of sectional area $a$, and with its base at $Q$ at a distance $h$ from P. If the molecular force between two molecules of the liquid is $3 \mathrm{~A} m^{2} / r^{4}$, then, expressing the

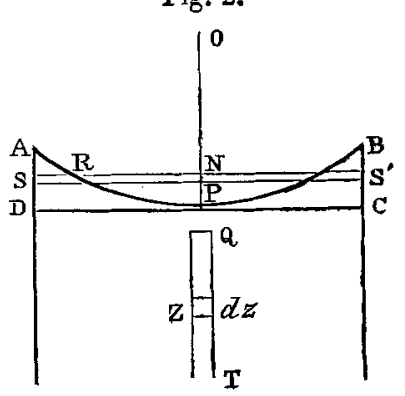


force parallel to $\mathrm{OT}$ exerted by a ring of radius $r$ about $\mathrm{N}$, and of thickness $d l$ on an element $d z$ of the rod, we get, if $\mathrm{ON}=l$ and $\mathrm{OZ}=z$,

$$
3 \mathrm{~A} a \bar{\rho}^{2} \frac{d z d l 2 \pi r d r}{\left\{r^{2}+(z-l)^{2}\right\}^{2}} \frac{z-l}{\left\{r^{2}+(z-l)^{2}\right\}^{\frac{1}{2}}} .
$$

Integrate with respect to $r$ from $N R$ to $N S=C$ the radius of the tube, with respect to $z$ from $O Q$ to a limit which we may indicate by the symbol $\infty$, and with respect to $l$ from the limit $h$, which is the distance $O$ from the plane $A B$ up to $R$, which is $O P$ the radius of the surface, and we have the desired expression :

$$
\int_{h}^{\mathrm{R}} \int_{\mathrm{R}+b}^{\infty} \int_{\sqrt{\mathrm{R}^{2}-l^{2}}}^{\sqrt{\mathrm{R}^{2}-c^{2}}} 2 \pi 3 \mathrm{~A} a \bar{\rho}^{2} \frac{(z-l) d z d l r d r}{\left\{r^{2}+(z-l)^{2}\right\}^{\frac{3}{2}}} .
$$

The integral evaluated becomes

$$
\begin{aligned}
& 2 \pi \mathrm{A} \bar{\rho}^{2} a\left\{\frac{\left(\mathrm{R}^{2}+(\mathrm{R}+b)^{2}-2(\mathrm{R}+b) h\right)^{\frac{1}{2}}-b}{\mathrm{R}+b}\right. \\
& \left.+\log \frac{b+\sqrt{c^{2}+b^{2}}}{\mathrm{R}+b-h+\sqrt{c^{2}+(\overline{\mathrm{R}}+b-h)^{2}}}\right\} \text {. }
\end{aligned}
$$

Expanded in powers of $b$ as far as 1st, and with $s$ put for $\mathrm{R}-h$, this becomes

$$
2 \pi \mathrm{A} a \bar{\rho}^{2}\left[\log \frac{c}{s+\sqrt{2 s \mathrm{R}}}+e\left\{\frac{1}{c}-\frac{1}{\mathrm{R}}+\frac{\sqrt{2 s \mathrm{R}}+\mathrm{R}-s-\frac{1}{2} s \sqrt{\overline{2 s}}}{\mathrm{R}(\overline{\mathrm{R}}+\sqrt{2 s \mathrm{R}})}\right\}\right] .
$$

Let us call the angle of contact of meniscus-surface with tube $\theta$. We know, from the usual theory of capillarity verified by experiment, that $\theta$ is constant; and this result holds in the same manner for the law of the inverse fourth power, so that, in the above expression, $c / \mathrm{R}$, which $=\cos \theta$, $s / \mathrm{R}$, which $=1-\sin \theta$, are both constant ; and therefore the first term is independent of the curvature of the meniscus, while the coefficient of $e$ may be written

$$
2 \pi \mathrm{A} a \bar{\rho}^{2}\left\{\frac{1}{\mathrm{R} \cos \theta}-\frac{1}{\mathrm{R}}+\frac{\sqrt{2(1-\sin \theta})+\sin \theta-\frac{1}{\sqrt{2}}(1-\sin \theta)^{\frac{3}{2}}}{\mathrm{R}\{1-\sin \theta+\sqrt{2(1-\sin \theta)}\}}\right\}
$$

We leave out of count the constant term as not entering into the question of capillary action, and compare our last expression with the expression which we get for the capillary pressure on the column QT, due to a tension $\alpha$ per unit width 
of the surface-layer, namely $2 \mathrm{~T} a / \mathrm{R}$. We see that this tension, or the energy per unit surface, is

$\alpha=\pi \bar{\rho}^{2} A e\left\{\frac{1}{\cos \theta}-1+\frac{\sqrt{2(1-\sin \theta)}+\sin \theta-\frac{1}{\sqrt{2}}(1-\sin \theta)^{\frac{3}{2}}}{1-\sin \theta+\sqrt{2(1-\sin \theta)}}\right\}$.

This equation is founded on the assumption that the capillary tube is so small that the meniscus-surface may be considered to be a portion of a sphere. When this is not close enough to the truth we shall require a more extended investigation of the necessary form of the meniscus, but for present purposes the above will suffice. It gives us an important relation between the parameter $A$ and the surface-tension, but one involving the unknown average density $\bar{\rho}$ of the surface-film, and the angle $\theta$ which is difficult to determine. But, however, as $\theta$, if not zero for all ordinary liquids, is small, we will assume it to be zero, so that the bracketed expression above reduces to $\frac{1}{2+\sqrt{2}}$. And as in the first case we desire only relative values of $A$ for a number of substances, we cannot go far wrong in assuming that, at their boiling-points, the ratios of the mean density of the surface-film of different liquids to the ordinary densities are approximately the same; we will therefore write $\bar{\rho} \propto \rho$, and reduce our equation above to the form $\alpha \propto \mathrm{A} \rho^{2} e$.

Now it is natural to suppose that, in different liquids, $e$ is proportional to the mean distance apart of the molecules in the surface-film, that is to the cube root of the molecular domain (usually called the molecular volume);

$$
\therefore e \propto \sqrt[3]{m / \rho} \propto \sqrt[3]{m / \rho}:
$$

so that finally $k \mathrm{~A} \rho^{\frac{1}{3}} m^{\frac{1}{3}}=\alpha$, where $k$ is a constant approximately the same for all liquids if the tension $\alpha$ is measured at the boiling-point; but the strict equation for any one substance is

$A \bar{\rho} \bar{\rho}^{\frac{5}{3}} m \propto \bar{s}^{\frac{1}{s}} \propto \alpha$.

Eötvös's result is that

$$
\frac{d}{d t}\left\{\alpha\left(\frac{m}{\rho}\right)^{\frac{2}{3}}\right\}=\cdot 227
$$

for most liquids, which, according to our equation above, gives

$$
\frac{d}{d t}(k \mathrm{~A} m \rho)=\cdot 227
$$

or

$$
k \mathrm{~A} m \frac{d \rho}{d t}=\cdot 227
$$


This equation contains two important consequences :-

First. The parameter of molecular force varies inversely as the product of molecular weight and modulus of dilatation. [Mendelejeff calls $d \rho / d t$ the modulus of dilatation, and shows that, for a given liquid, it is approximately independent of temperature when measured at low pressures (one atmosphere).]

Second. The rate of variation of the translatory kinetic energy of the molecules of most liquids with temperature is the same, if that rate is measured at a constant low pressure, such as that of one atmosphere. This second consequence flows from the above equation in the following manner. It is shown in my previous paper (Phil. Mag. July 1887) that the virial of the mutual attractions of the molecules in unit mass of a body, according to the law of the inverse fourth power, is $\pi \mathrm{A} \rho \log \mathrm{L} / a$; where $\mathrm{L}$ is a sensible length such as the cube root of the volume of the unit mass, and $a$ is a length approximately proportional to the cube root of the molecular domain (usually called molecular volume). L/a is so large a number that $\log \mathrm{L} / a$ may be regarded as constant within the limits of present experimental possibility in the variation of $\mathrm{L}$ and $a$.

Now Clausius' equation of the virial applied to gases is $\frac{3}{2} p v+$ internal virial $=$ translatory kinetic energy.

In the case of liquids under a small constant pressure, $p v$ is negligible in comparison with the other terms of the equation; so that for liquids at low pressures the equation assumes the simple form

$$
\text { internal virial }=\text { translatory kinetic energy ; }
$$

while for gases at low pressures, the internal virial being negligible, it assumes the form

$$
\frac{3}{2} p v \text { or external virial }=\text { translatory kinetic energy. }
$$

In the equation for liquids let us replace the internal virial by its value $3 \pi \mathrm{A} \rho \log \mathrm{L} / a$, and denote the kinetic energy of translation of the molecules in unit mass by $\mathrm{E}$; then

$$
\begin{aligned}
3 \pi \mathrm{A} \rho \log \mathrm{L} / a & =\mathrm{E}, \\
\therefore 3 \pi \mathrm{A} m \log \frac{\mathrm{L}}{a} \frac{d \rho}{d t} & =\frac{d(m \mathrm{E})}{d t} .
\end{aligned}
$$

But $\mathrm{A} m \frac{d \rho}{d t}$ is the same for most liquids,

$$
\therefore \frac{d(m \mathrm{E})}{d t}
$$


or the temperature rate of variation of the translatory kinetic energy of a molecule, is the same for all molecules when compared as the constituents of liquids at low constant pressure.

This is an important result in molecular dynamics; its similarity to the law of Dulong and Petit as to the constancy of the molecular heats of the elements is obvious and immediately suggestive, while its bearing on the physical meaning of temperature is worth noting. It must not be confused with the assumption made by many continental writers on thermodynamics and molecular physics, that the absolute temperature of a mass is a measure of the kinetic energy of translation of each molecule in the mass, no matter what the state of the mass be ; this assumption gives $m \mathrm{E} \propto \mathrm{T}$, and therefore $d(\mathrm{ME}) / d t$ is constant for all substances and for all states of each substance. But the result given above holds only when the external virial is negligible in comparison with the internal; in fact the difference botween the two views may be shown thus:-According to the arguments of this paper, we can write $d(m \mathrm{E}) / d t=\mathrm{P}$ when the external virial is negligible, while $d(m \mathrm{E}) / d t=\mathrm{Q}$ when the internal virial is negligible, $\mathrm{P}$ and $\mathrm{Q}$ having possibly different values; while, according to the assumption mentioned above, $\mathrm{P}$ is considered equal to Q. Again integrating, we get

and

$$
m \mathrm{E}=\int \mathrm{P} d t+\mathrm{M} \text { for the first case, }
$$

$$
m \mathrm{E}=\int \mathrm{Q} d t+\mathrm{N} \text { for the second case, }
$$

the constants of integration being possibly different; while, according to the above assumption, the two constants are assumed to be the same, seeing that the translatory kinetic energy of a molecule, both of a liquid and of a vapour at the same temperature, is assumed to be the same and to be proportional to the absolute temperature.

Before proceeding to the determination of the values of $\mathbf{A}$ from Schiff's experimental results, it may be as well to discuss a certain old-standing difficulty in capillarity, namely the rapid rate at which capillary height and surface-tension diminish with rising temperature. According to the expression found above, the tension for a given substance varies as the $\frac{5}{3}$ power of the average density of the film, $\alpha \propto \rho^{-\frac{5}{3}}$. Now C. Schall has shown (Berichte der deutsch. chem. Gesell. xiv. p. 555) that approximately the surface-tension for several liquids varies as the $\frac{8}{3}$ power of the density; although the theoretical arguments by which he endeavours to establish this relation as a consequence of the Newtonian law are not 
valid, still $\alpha \propto \rho^{\frac{8}{3}}$. The seeming discordance between these two values for $\alpha$ corresponds to the old difficulty in the early days of capillary theory, when Laplace's simplification of imagining the surface-density to be the same as the bodydensity was adopted, and the surface-tension was found experimentally to diminish much more rapidly with temperature than his theory indicated. But if we take account of the possibility of a markedly different density in the surface from that in the body, and recognize the corresponding difference of stress in the two regions, we shall be prepared to imagine that, approximately, $\rho^{\frac{5}{3}} \propto \rho^{\frac{8}{8}}$, which means that the rate of variation of the surface-density with temperature is more rapid than that of body-density. And this is a necessary corollary of our concession of a marked difference of density and stress in the two regions; for Van der Waals has accustomed us to the idea of a difference of stress, of the order of magnitude of 1000 atmospheres ; and the recent reseurches of Amagat, on compressibility at higher pressures and its variation with temperature, have shown that the coefficient of expansion of æether under one atmosphere between $0^{\circ}$ and $50^{\circ}$ is 0017 , while under 3000 atmospheres it is $\cdot 00056$, so that an increase in stress of 3000 atmospheres diminishes the coefficient to one third of its ordinary value. Thus then we see that, in a general way, the old difficulty is really a very direct confirmation of the great difference of stress and density in the surface-film and body of a liquid; and moreover experiment shows that heating the film alone produces a much more pronounced effect than heating the lower liquid only in a capillary tube.

We can now proceed to determine the values of the parameters $k \mathrm{~A}$ for a large number of compounds by applying the equation $\alpha=k \mathrm{~A} \rho^{\frac{5}{3}} m^{\frac{1}{3}}$ to Schiff's results.

The following table is devoted to the consideration in the first case of the values of $k \mathrm{~A}$ for the isomeric forms of the compound ethers of the general formula $\mathrm{C}_{n} \mathrm{H}_{2 n} \mathrm{O}_{2}$. For the sake of brevity the basic radicals methyl, ethyl, \&c. are denoted by I, II, \&c., and the basic radicals in ascending order by $1,2,3, \& c$. ; so that I 1 stands for methyl formiate, II 3 for ethyl propionate, and so on. Schiff's values of $\alpha$ are given in terms of grammes weight per linear metre, so that $k \mathrm{~A}$ in the following tables is given in corresponding units. The acids $\mathrm{C}_{n} \mathrm{H}_{2 n} \mathrm{O}_{2}$ are omitted from the table because Eötvös finds that they do not obey his law, and their anomalous vapour-densities are well known. 
Values of $k \mathrm{~A} \times 1000$.

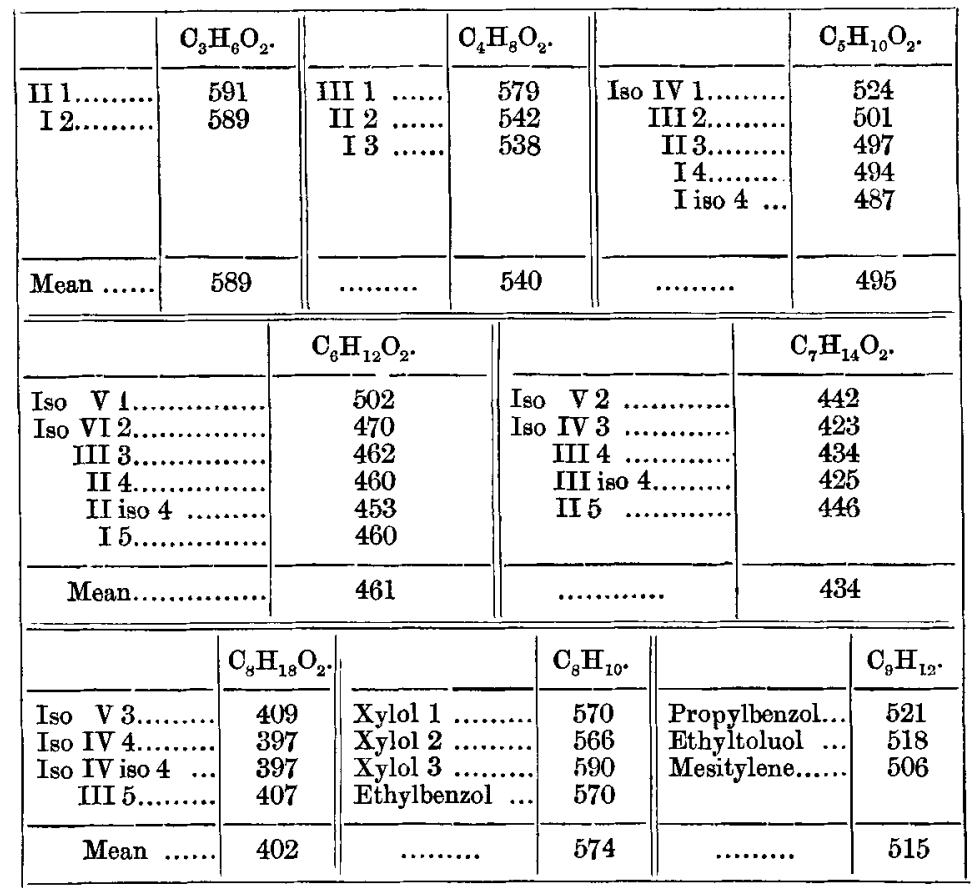

The mean values are taken with the formiates excluded because they depart so markedly from the rest, the formiates evidently tending towards the irregularity of the acids. The mean values are introduced into the following table, in which the values of $k \mathrm{~A}$ are arranged according to descending order. The second column contains the formulæ with $\mathrm{O}^{\prime}$ to indicate $O$ connected with two other atoms, $O^{\prime \prime}$ to indicate $O$ connected with a single atom. Inspection of the table shows that the number of $\mathrm{H}$ atoms in the molecule exercises no appreciable influence on the value of $k \mathrm{~A}$, and that $\mathrm{O}^{\prime}$ produces the same effect as $2 \mathrm{C}$, and $\mathrm{O}^{\prime \prime}$ as $3 \mathrm{C}$. In the third column there is placed the number $n$ of $\mathrm{C}$ atoms that the molecule is equivalent to, which I shall call its parameter equivalent. In the fifth column the mean value of $k \mathrm{~A}$ for a given value of $n$, or $\overline{k A}$; and the last column contains the values of $\overline{k \mathrm{~A}} n^{\frac{2}{3}}$. 


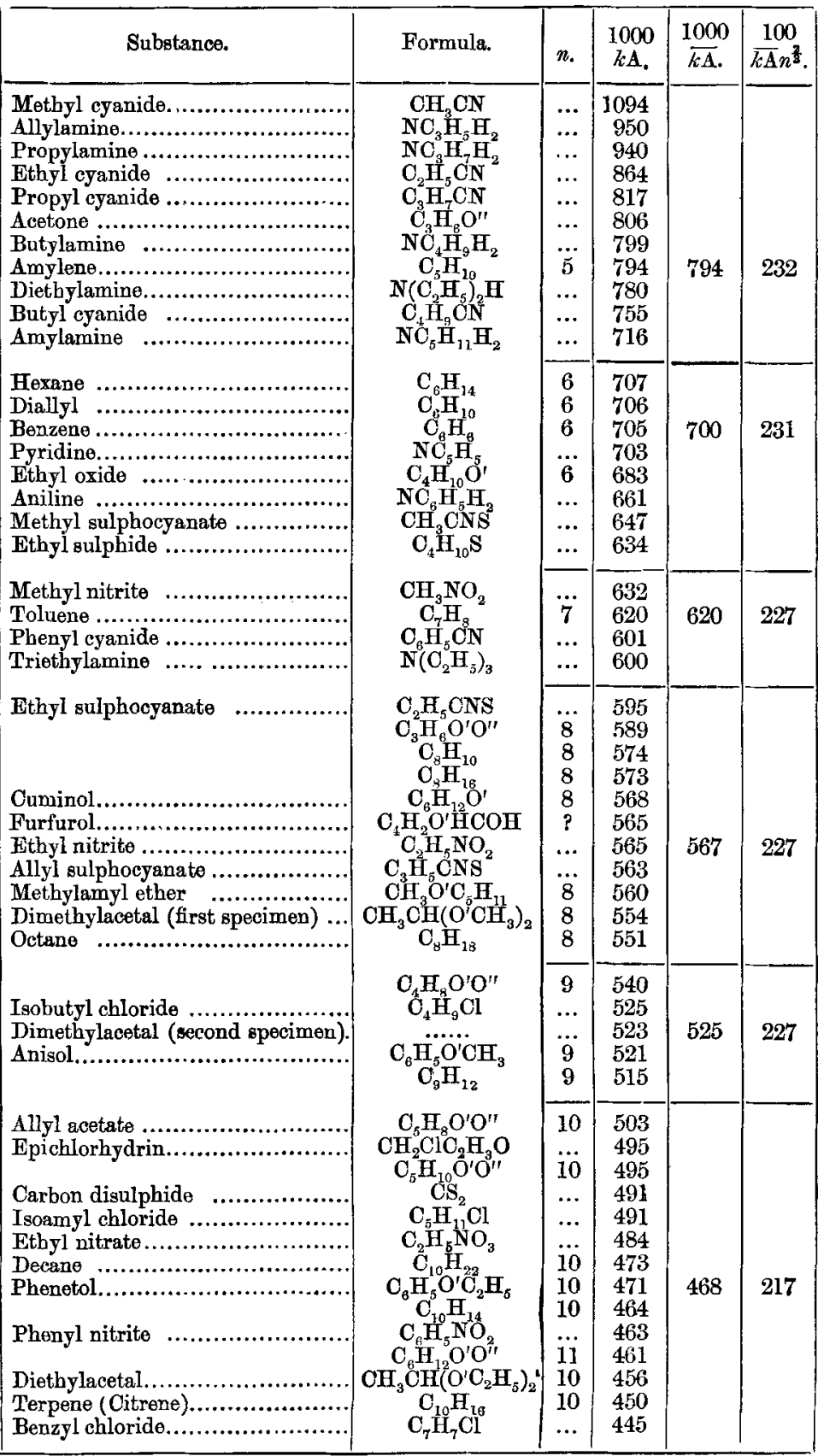


Table (continued).

\begin{tabular}{|c|c|c|c|c|c|}
\hline Substance. & Formula. & $n$. & $\begin{array}{r}1000 \\
k \mathrm{~A} .\end{array}$ & $\frac{1000}{\overline{k A}}$ & $\frac{1000}{k \mathrm{kA} n}$ \\
\hline 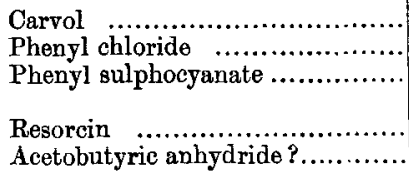 & $\begin{array}{c}\mathrm{C}_{10} \mathrm{H}_{14} \mathrm{O} \\
\mathrm{C}_{6} \mathrm{H}_{5} \mathrm{Cl} \\
\mathrm{C}_{6} \mathrm{H}_{5} \mathrm{CNS}^{\prime} \\
\mathrm{C}_{7} \mathrm{H}_{14} \mathrm{OO}^{\prime} \\
\mathrm{C}_{6} \mathrm{H}_{2}\left(\mathrm{O}^{\prime} \mathrm{HCH}_{3}\right)_{2} \\
\mathrm{C}_{2} \mathrm{H}_{3} \mathrm{O}_{3}^{\prime} \mathrm{O}_{4} \mathrm{H}_{7}\end{array}$ & $\begin{array}{l}12 ? \\
\cdots \\
12 \\
12 \\
12 ?\end{array}$ & $\begin{array}{l}444 \\
440 \\
434 \\
431 \\
431\end{array}$ & 435 & 228 \\
\hline 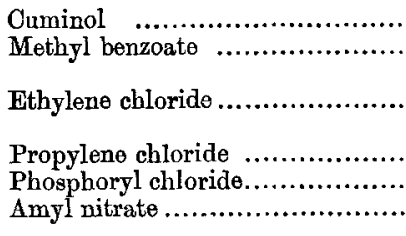 & $\begin{array}{c}\mathrm{C}_{70} \mathrm{H}_{72} \mathrm{O}^{\prime \prime} \\
\mathrm{C}_{6} \mathrm{H}_{5} \mathrm{CO}^{\prime} \mathrm{O}^{\prime \prime} \mathrm{CH}_{3} \\
\mathrm{O}_{7} \mathrm{H}_{7} \mathrm{Cl} \\
\mathbf{O}_{2} \mathrm{H}_{4} \mathrm{Cl}_{2} \\
\mathrm{O}_{8} \mathrm{H}_{16} \mathrm{O}^{\prime} \mathrm{O}^{\prime \prime} \\
\mathbf{C}_{3} \mathrm{H}_{6} \mathrm{Cl}_{2} \\
\mathrm{POCl}_{2} \\
\mathrm{C}_{5} \mathrm{H}_{11} \mathrm{NO}_{3}\end{array}$ & $\begin{array}{l}13 \\
13 \\
\cdots \\
\dddot{13} \\
\cdots \\
\cdots \\
\cdots\end{array}$ & $\begin{array}{l}422 \\
417 \\
417 \\
412 \\
402 \\
390 \\
388 \\
388\end{array}$ & 414 & 229 \\
\hline 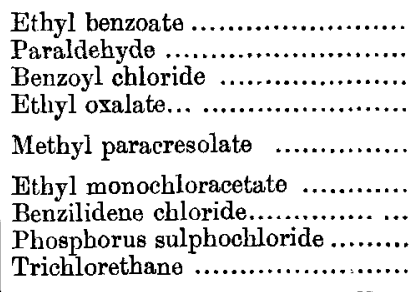 & 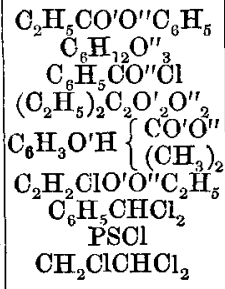 & $\begin{array}{l}14 \\
15 \\
\ldots \\
16 \\
\cdots \\
\cdots \\
\cdots\end{array}$ & $\begin{array}{l}387 \\
379 \\
373 \\
365 \\
362 \\
359 \\
357 \\
356\end{array}$ & $\begin{array}{l}387 \\
379 \\
\\
365\end{array}$ & $\begin{array}{l}225 \\
231 \\
\\
232\end{array}$ \\
\hline & & & Mea & lue & 228 \\
\hline
\end{tabular}

This table shows that, although the values of $k \mathrm{~A}$ do not change at a bound on passing from one value of $n$ to another, yet the mean value of $k \mathrm{~A}$ taken for a given value of $n$ found in the manner indicated varies closely inversely as the twothirds power of $n$ or $k \mathrm{~A}=228 / n^{\frac{3}{3}}$.

A study of the table shows that the compounds containing chlorine possess such values of $k \mathrm{~A}$ as would make $\mathrm{Cl}$ equal to $5 \mathrm{C}$ or $6 \mathrm{C}$. With regard to $\mathrm{N}$ in the cyanides, its value appears to diminish as the molecular weight of the compounds into which it enters increases; in the amines, on the other hand, $\mathrm{N}$ can with considerable accuracy be put equal to $\mathrm{C}$; or if we solve strictly for $n$ by the equation above, the value deduced for $\mathrm{N}$ is $\cdot 8 \mathrm{C}$. In the nitrites $\mathrm{NO}_{2}=5 \cdot 6 \mathrm{C}$ and as $\mathrm{O}_{2}=\mathrm{O}^{\prime} \mathrm{O}^{\prime \prime}=5 \mathrm{C}, \mathrm{N}$ in the nitrites $=6 \mathrm{C}$. In the nitrates $\mathrm{NO}_{3}$ in the mean has the value $8 \cdot 6 \mathrm{C}$; and as $\mathrm{O}_{3}=\mathrm{O}^{\prime} \mathrm{O}_{2}{ }^{\prime \prime}=8 \mathrm{C}$, the value of $\mathrm{N}$ in the nitrates is $6 \mathrm{C}$.

The small influence of the hydrogen atom in the molecule is shown by the fact that in the previous table the number of $\mathrm{H}$ atoms has been ignored; with a more accurate treatment the 
actual value of $H$ may be determined; but for the present, as a general result, we regard $H$ as negligible.

If we regard the number $n$ as measuring the actual volume of the molecule, and if we consider molecules to have similar shapes, then $n^{\frac{2}{3}}$ measures the surface of the molecule; and we can say that the parameter A varies inversely as the surface of the molecule. This interpretation is partially justified by the consideration of certain results in molecular refraction. Let $i$ be the index of refraction of a substance whose molecule occupies a domain $u$, but actually fills only a volume $U$, and let $I$ be the index of refraction of the matter of the molecule, then I have shown in my paper on Molecular Refraction that $(i-1) u=(\mathrm{I}-1) \mathrm{U}$, which is Gladstone's law. Now Landolt, Gladstone, and Brühl have shown that in general $(i-1) u$ for a molecule is the sum of certain quantities known as the refraction-equivalents characteristic of the different atoms, but that there are pronounced exceptions : thus, when an atom possesses two valencies, it has two corresponding refractionequivalents. The same results hold therefore for $(\mathrm{I}-1) \mathrm{U}$; thus, if an atom has different effective valencies, it contributes different amounts to the value of $(I-1) U$ in a molecule according to its valency; thus either I or $\mathrm{U}$, or both I and U, alter with the effective valency. Now we have considered $n$ to be proportional to $\mathrm{U}$; and we have found $n$ in the case of oxygen to vary with valency, so that the optical argument for a possible variation of $U$ with valency agrees with the variation in the value of $n$ which we have found. It is to be observed here that this effect of change of valency in changing $n$ may tend to obscure the value of $\mathrm{H}$ as compared with $\mathrm{C}$; for in most cases a reduction in the number of $\mathrm{H}$ atoms is accompanied by a change of valency of the $\mathrm{C}$ atoms. In the study of molecular refraction we find in a large number of cases that the reduction of molecular refraction caused by the expulsion of $\mathrm{H}$ atoms for a molecule is almost exactly counterbalanced by the increased refraction-equivalent of $\mathrm{C}$ due to changed valency.

In the previous table the value obtainable from Schiff's data for certain organic bromides and iodides have been omitted, because of their exceptional character. The following table contains the values of $k \mathrm{~A}$ for various substances.

Values of $k \mathrm{~A} \times 1000$.

$\begin{array}{cccccc}\mathrm{Br}_{2} & \mathrm{C}_{4} \mathrm{H}_{5} \mathrm{Br} & \mathrm{C}_{3} \mathrm{H}_{7} \mathrm{Br} & \mathrm{C}_{3} \mathrm{H}_{7} \mathrm{Br} \text { (Iso) } & \mathrm{C}_{3} \mathrm{H}_{5} \mathrm{Br} & \mathrm{C}_{4} \mathrm{H}_{9} \mathrm{Br} \\ 104 & 259 & 269 & 270 & 269 & 271 \\ & \mathrm{C}_{5} \mathrm{H}_{11} \mathrm{Br} & \mathrm{C}_{6} \mathrm{H}_{5} \mathrm{Br} & \mathrm{C}_{7} \mathrm{H}_{7} \mathrm{Br} & \mathrm{C}_{2} \mathrm{H}_{4} \mathrm{Br}_{2} & \mathrm{C}_{3} \mathrm{H}_{6} \mathrm{Br}_{2} \\ 270 & 247 & 247 & 149 & 149\end{array}$


Law of Molecular Force.

$\begin{array}{cccccc}\mathrm{CH}_{3} \mathrm{I} & \mathrm{C}_{2} \mathrm{H}_{5} \mathrm{I} & \mathrm{C}_{3} \mathrm{H}_{7} \mathrm{I} & \mathrm{C}_{3} \mathrm{H}_{7} \mathrm{I}(\mathrm{Iso}) & \mathrm{C}_{3} \mathrm{H}_{5} \mathrm{I} & \mathrm{C}_{4} \mathrm{H}_{9} \mathrm{I} \\ 150 & 159 & 171 & 170 & 170 & 176 \\ & & \mathrm{C}_{5} \mathrm{H}_{11} \mathrm{I} & \mathrm{C}_{6} \mathrm{H}_{5} \mathrm{I} & & \\ & & & 178 & 168 & \end{array}$

In the case of bromides containing one atom of $\mathrm{Br}$ we see that the value of $k \mathrm{~A}$ is stationary, or increases a little with increasing molecular weight, while in the case of the iodides the same phenomenon is more pronounced, the value increases with addition of $\mathrm{CH}_{2}$ instead of diminishing, as it does in compounds containing $\mathrm{C}, \mathrm{H}, \mathrm{O}, \mathrm{N}$, and $\mathrm{Cl}$. The exceptional character of these results is not surprising when we consider the large masses of the $\mathrm{Br}$ and $\mathrm{I}$ atoms. The refraction-equivalents of the organic bromides and iodides are quite regular; so that there is no ground for thinking that the volume of a $\mathrm{Br}$ or of an I atom varies according to the number of carbon atoms with which it is united; but the surface of a molecule containing $\mathrm{Br}$ or I united to an organic radical may perhaps no longer be considered to be proportional to the two-thirds power of the molecule on account of the pronounced want of symmetry in structure due to the preponderant atomic mass of $\mathrm{Br}$ and $\mathrm{I}$. With this in view, and remembering that if $\mathrm{Br}$ and I parameter-equivalents are large, as the preceding table shows that they must be, then $228 / n^{\frac{2}{3}}$ would vary slowly with the slight variation in $n$ produced by the introduction into the molecule of a few $\mathrm{C}$ atoms ; the effect, therefore, of dyssymmetry in the building up of the organic bromides and iodides would not require to be very great to explain the exceptional character of $k \mathrm{~A}$ in them.

In conclusion, we will see what light the law of the inverse fourth power throws on Waterston's law. If for a moment we ignore thermodynamical considerations and regard the evaporation of a liquid from the purely mechanical point of view as a change of a system of molecules attracting according to the inverse fourth power from a configuration where the density is $\rho$ to one in which it is $\sigma$, then, as shown in my former paper (Phil. Mag. July 1887), the mutual potential energy of the molecules in the two configurations is $2 \pi \mathrm{A} \rho \log \mathrm{L} / a$ and $2 \pi A \sigma \log \mathrm{L} / a$ respectively. If, then, for the moment we consider the latent heat-vaporization as equivalent to this change of potential energy and call the latent heat $\lambda$, we have

$$
\lambda=2 \pi \mathrm{A}\{\rho-\sigma\} \log \frac{\mathrm{L}}{a} .
$$

Now, except in the neighbourhood of the critical point, we can neglect $\sigma$, the density of the vapour, in comparison with $\rho$, 
that of the liquid, and can write

$$
\lambda=2 \pi \operatorname{A} \rho \log \frac{\mathrm{L}}{a} \text {. }
$$

But our equation for the surface-tension gives

$$
\begin{gathered}
\alpha=k \mathrm{~A} \rho^{\frac{3}{3}} m^{\frac{1}{3}} ; \\
\therefore \frac{m \lambda}{\frac{\alpha \frac{2}{3}^{\frac{2}{3}}}{\rho^{\frac{3}{3}}}}=\frac{2 \pi \log \frac{\mathrm{L}}{a}}{k}=\text { constant. }
\end{gathered}
$$

This is Waterston's law. Now according to thermodynamical principles the latent heat of evaporation without performance of external work is

$$
\int_{\frac{1}{\sigma}}^{\frac{1}{\rho}} \theta \frac{\partial p}{\partial \theta} d v
$$

where $\theta$ is the absolute temperature at which evaporation takes place. This cannot be evaluated till we know the characteristic equation for fluids, which bridges in a complete manner the gap between the liquid and the gaseous states. But if we make the assumption that the latent heats of all fluids at their boiling-points are approximately the same fraction of the change of potential energy of the molecules due to evaporation, then Waterston's law would still be an approximate deduction from the law of the inverse fourth power. And this assumption is a natural one, seeing that Ramsay and Young have pointed out that the external work done during evaporation at the ordinary boiling-point is approximately the same fraction of the total latent heat for all liquids; our assumption is, that the fraction of the latent heat due to thermodynamical adjustment is for all liquids the same fraction of the change of mechanical energy. However, that the assumption is not a sound one is shown by the roughness of the approach to constancy of the following values of $\frac{m \lambda \rho^{\frac{2}{3}}}{\alpha m^{\frac{2}{3}}}$. The alcohols and acids of the fatty series are omitted because of their not following Eötvös's law.

$$
\begin{array}{cccccccc}
\mathrm{C}_{5} \mathrm{H}_{10} & \mathrm{C}_{6} \mathrm{H}_{6} & \mathrm{C}_{10} \mathrm{H}_{16} & \mathrm{CHCl}_{3} & \mathrm{CCl}_{4} & \mathrm{C}_{2} \mathrm{H}_{5} \mathrm{Br} & \mathrm{C}_{2} \mathrm{H}_{5} \mathrm{I} & \mathrm{C}_{2} \mathrm{H}_{4} \mathrm{Br}_{2} \\
6 \cdot 7 & 6 \cdot 2 & 4 \cdot 5 & 5 \cdot 8 & 6 \cdot 2 & 6 \cdot 1 & 5 \cdot 9 & 6 \cdot 7 \\
\mathrm{C}_{5} \mathrm{H}_{11} \mathrm{Cl} & \mathrm{C}_{5} \mathrm{H}_{11} \mathrm{Br} & \mathrm{C}_{5} \mathrm{H}_{11} \mathrm{I} & \mathrm{CH}_{3} \mathrm{I} & \mathrm{C}_{3} \mathrm{H}_{6} \mathrm{O} \text { (acetone) } \\
6 \cdot 9 & 6 \cdot 1 & 4 \cdot 9 & 6 \cdot 7 & 4 \cdot 7 &
\end{array}
$$




$\begin{array}{ccccc}\mathrm{CCl}_{3} \mathrm{COH} & \left(\mathrm{C}_{2} \mathrm{H}_{5}\right)_{2} \mathrm{C}_{2} \mathrm{O}_{4} & \left(\mathrm{CH}_{3} \mathrm{CO}\right)_{2} \mathrm{O} & \mathrm{O}_{3} \mathrm{H}_{6} \mathrm{O}_{2} & \mathrm{C}_{4} \mathrm{H}_{8} \mathrm{O}_{2} \\ 5 \cdot 3 & 4 \cdot 4 & 6 \cdot 7 & 5 \cdot 5 & 5 \cdot 4 \\ & & & \text { Mean. } & \text { Mean. }\end{array}$

\section{$\mathrm{C}_{5} \mathrm{H}_{10} \mathrm{O}_{2} \quad \mathrm{C}_{6} \mathrm{H}_{12} \mathrm{O}_{2} \quad \mathrm{C}_{7} \mathrm{H}_{14} \mathrm{O}_{2} \quad \mathrm{C}_{8} \mathrm{H}_{16} \mathrm{O}_{2}$ $\begin{array}{llll}5 \cdot 3 & 6.0 & 6 \cdot 5 & 6.9\end{array}$ \\ Mean. Mean. Mean. Mean.}

The previous numbers show that Waterston's law, while representing a remarkable attempt at the time it was announced to connect latent heat, surface-tension, and molecular volume, is far from being verified by experimental data; and they show the danger of pushing the idea of "correspondence" too far. In fact, it would appear that the manner in which Eötvös deduces his law theoretically from the principle of " correspondence" only happens by chance to give a rigorous law, as the same principle applied to latent heats and surface-energy leads to a law so far from rigorous as Waterston's has been shown to be.

In the preceding pages we have argued from Eötvös's empirical law as basis ; but probably, when the dynamical meaning of temperature in liquids has been made clear, Eötvös's law will be deduced in the following manner.

The temperature of a liquid subjected to small constant pressure is such a property of the molecule of the liquid, that its rate of variation with the translatory kinetic energy of the molecule is the same for all molecules; but as external virial is negligible under these circumstances, we have the rate of variation with temperature of internal virial multiplied by molecular mass proportional to the rate of variation of the translatory kinetic energy of the molecule, which is the same for all molecules. But according to the law of the inverse fourth power, internal virial multiplied by the mass of the molecule is proportional to surface-tension multiplied by the two-thirds power of the molecular domain, whence Eötvös's law follows, that the rate of variation with temperature of the product of surface-tension and the two-thirds power of the molecular domain is the same for nearly all liquids.

In the present paper we have been occupied only with attractions between similar molecules; and there is too little experimental material to allow of our testing any hypothesis as to the parameter of molecular attraction in the case of dissimilar ones; but it seems probable that the parameter will be inversely proportional to the product of the square roots of the surfaces of the two molecules. 